\title{
A further study on the coupled Allen-Cahn/Cahn-Hilliard equations
}

Jiaqi Yang ${ }^{1 *}$ and Changchun Liu ${ }^{1}$

"Correspondence:

yangjq18@mails.jlu.edu.cn

${ }^{1}$ Department of Mathematics, Jilin

University, Changchun, China

\begin{abstract}
In this paper, we will show that solutions of the initial boundary value problem for the coupled system of Allen-Cahn/Cahn-Hilliard equations continuously depend on parameters of the system, and under some restrictions on the parameters all solutions of the initial boundary value problem for Allen-Cahn/Cahn-Hilliard equations tend to zero with an exponential rate as $t \rightarrow \infty$.
\end{abstract}

MSC: Primary 35M30; secondary 35B35; 35B40

Keywords: Decay estimate; Continuous dependence; Allen-Cahn/Cahn-Hilliard equations

\section{Introduction}

In this paper, we consider the following Allen-Cahn/Cahn-Hilliard system:

$$
\begin{aligned}
& \partial_{t} A-\alpha A-\partial_{x}^{2} A+k|A|^{2} A=A h, \quad x \in(0, l), t>0, \\
& \partial_{t} h+|A| \partial_{x} h=m \partial_{x}^{2} \mu, \quad x \in(0, l), t>0, \\
& A(x, 0)=A_{0}(x), \quad h(x, 0)=h_{0}(x), \quad x \in(0, l), \\
& A(0, t)=A(l, t)=\overrightarrow{0},\left.\quad \partial_{x} h(x, t)\right|_{x=0, l}=\left.\partial_{x}^{3} h(x, t)\right|_{x=0, l}=0, \quad t>0,
\end{aligned}
$$

where $\mu=f^{\prime}(h)-\gamma \partial_{x}^{2} h, f^{\prime}(h)=h^{3}-h$, and $\overrightarrow{0}$ is a zero vector of $R^{N}, m k>\frac{5}{4}, m, k, \alpha>0$ are given numbers, $A(x, t)=\left(A_{1}(x, t), \ldots, A_{N}(x, t)\right)$ is the unknown vector function, $h(x, t)$ is the unknown scalar function, $A_{0}(x)$ and $h_{0}(x)$ are given initial data.

System (1.1)-(1.4) was introduced to model simultaneous order-disorder and phase separation in binary alloys on a BCC lattice in the neighborhood of the triple point [1]. Here, $h$ denotes the concentration of one of the components, while $A$ is an order parameter. The Allen-Cahn equation and the Cahn-Hilliard equation have been intensively studied [2-5]. Miranville, Saoud, and Talhouk [5] studied the long time behavior, in terms of finite-dimensional attractors, of a coupled Allen-Cahn/Cahn-Hilliard system. In particular, they proved the existence of an exponential attractor and, as a consequence, the existence of a global attractor with finite fractal dimension. Çelebi and Kalantarov [6] proved the decay of solutions and structural stability for the coupled Kuramoto-SivashinskyGinzburg-Landau equations.

(c) The Author(s) 2019. This article is distributed under the terms of the Creative Commons Attribution 4.0 International License (http://creativecommons.org/licenses/by/4.0/), which permits unrestricted use, distribution, and reproduction in any medium, provided you give appropriate credit to the original author(s) and the source, provide a link to the Creative Commons license, and indicate if changes were made. 
The large time behavior and the structural stability of solutions are important for the study of a higher-order parabolic system. Many papers have already been published to study the decay and the structural stability of solutions [7-9]. In this paper, we consider the asymptotic behavior of solutions and the continuous dependence of solutions for system (1.1)-(1.4). We are going to show the continuous dependence when the coefficient changes, which helps us to know whether a coefficient in the system can cause a large change in the solution.

The following is the main result of the paper.

\section{Theorem 1.1 If}

$$
\alpha<\lambda_{1} \quad \text { and } \quad \lambda_{1}\left(\gamma m-\frac{1}{2 \lambda_{1}^{2}}\right)-\frac{3 m}{2}>0,
$$

then all solutions of problem (1.1)-(1.4) tend to zero with an exponential rate as $t \rightarrow \infty$.

Theorem 1.1 implies that the concentration of one of the components and the order parameter will tend to zero as $t \rightarrow \infty$. Hence one of the components will disappear and the system will become disorder in a background point of view.

To prove Theorem 1.1, the basic a priori estimates are the $L^{2}$ norm estimates on $h$ and $\partial_{x} h$. The main difficulties are caused by the nonlinearity of both the diffusive and the convective factors in equation (1.2). To overcome such difficulty, we establish two new functionals $E_{1}(t)$ and $E_{2}(t)$. Our method is based on the global energy estimates and require some delicate local integral estimates.

This paper is arranged as follows. We first study a priori estimates in Sect. 2, and then establish the exponential decay of solution in Sect. 3. Subsequently, we discuss the continuous dependence results in Sect. 4.

\section{A priori estimates}

Similar to [10], we know that problem (1.1)-(1.4) has a unique global solution. The first step is to obtain a priori estimates of solutions of system (1.1), (1.2). Applying the operator $P^{2}$ to both sides of equation (1.2), here $P^{2}$ is the inverse operator of the operator $L=-\frac{d^{2}}{d x^{2}}$ with the domain of definition $D(L)=H^{2}(0, l) \cap H_{0}^{1}(0, l)$, we get the following problem:

$$
\begin{aligned}
& \partial_{t} A-\alpha A-\partial_{x}^{2} A+k|A|^{2} A=A h, \quad x \in(0, l), t>0, \\
& P^{2} \partial_{t} h+P^{2}\left(|A| \partial_{x} h\right)=-m \mu, \quad x \in(0, l), t>0, \\
& A(x, 0)=A_{0}(x), \quad h(x, 0)=h_{0}(x), \quad x \in(0, l), \\
& A(0, t)=A(l, t)=\overrightarrow{0}, \quad \partial_{x} h(0, t)=\partial_{x} h(l, t)=0, \quad t>0 .
\end{aligned}
$$

Multiplying equation (2.1) by $A$ and (2.2) by $h$ shows

$$
\frac{1}{2} \frac{d}{d t}\|A(t)\|^{2}-\alpha\|A(t)\|^{2}+\left\|\partial_{x} A(t)\right\|^{2}+k \int_{0}^{l}|A(x, t)|^{4} d x=\left(|A(t)|^{2}, h(t)\right)
$$

and

$$
\frac{1}{2} \frac{d}{d t}\|P h(t)\|^{2}+\int_{0}^{l}\left(|A(x, t)| \partial_{x} h(x, t)\right) P^{2} h(x, t) d x
$$




$$
=-m \int_{0}^{l}|h(x, t)|^{4} d x+m\|h(t)\|^{2}-\gamma m\left\|\partial_{x} h(t)\right\|^{2} .
$$

Adding the two resulting equations together, we obtain

$$
\begin{aligned}
\frac{1}{2} \frac{d}{d t}( & \left.\|A(t)\|^{2}+\|P h(t)\|^{2}\right)-\alpha\|A(t)\|^{2}+\left\|\partial_{x} A(t)\right\|^{2}+m \int_{0}^{l}|h(x, t)|^{4} d x \\
& +k \int_{0}^{l}|A(x, t)|^{4} d x+\gamma m\left\|\partial_{x} h(t)\right\|^{2} \\
= & m\|h(t)\|^{2}+\int_{0}^{l}|A(x, t)|^{2} h(x, t) d x-\int_{0}^{l}\left(|A(x, t)| \partial_{x} h(x, t)\right) P^{2} h(x, t) d x \\
\leq & m\|h(t)\|^{2}+\frac{1}{2 m} \int_{0}^{l}|A(x, t)|^{4} d x+\frac{m}{2}\|h(t)\|^{2}+\frac{1}{8 m} \int_{0}^{l}|A(x, t)|^{4} d x \\
& +\frac{m \lambda_{1}^{4}}{2} \int_{0}^{l}\left|P^{2} h(x, t)\right|^{4} d x+\frac{1}{2 \lambda_{1}^{2}} \int_{0}^{l}\left|\partial_{x} h(x, t)\right|^{2} d x \\
\leq & \frac{3 m}{2}\|h(t)\|^{2}+\frac{5}{8 m} \int_{0}^{l}|A(x)|^{4} d x+\frac{1}{2 \lambda_{1}^{2}}\left\|\partial_{x} h(t)\right\|^{2}+\frac{m}{2} \int_{0}^{l}|h(x, t)|^{4} d x,
\end{aligned}
$$

that is,

$$
\begin{aligned}
\frac{1}{2} \frac{d}{d t}\left(\|A(t)\|^{2}+\|P h(t)\|^{2}\right)+\left\|\partial_{x} A(t)\right\|^{2}+\frac{m}{2} \int_{0}^{l}|h(x, t)|^{4} d x \\
\quad+\frac{k}{2} \int_{0}^{l}|A(x, t)|^{4} d x+\left(\gamma m-\frac{1}{2 \lambda_{1}^{2}}\right)\left\|\partial_{x} h(t)\right\|^{2} \\
\leq \alpha\|A(t)\|^{2}+\frac{3}{2} m\|h(t)\|^{2} .
\end{aligned}
$$

Due to the Cauchy-Schwarz inequality, we have

$$
\|h\|^{2}=\left(P^{-1} h, P h\right) \leq\left\|P^{-1} h\right\|\|P h\| \leq \varepsilon\left\|\partial_{x} h\right\|^{2}+C_{1}(\varepsilon)\|P h\|^{2} .
$$

Choosing $\varepsilon=\frac{\gamma}{3}-\frac{1}{3 m \lambda_{1}^{2}}$ and $C_{1}(\varepsilon)=\frac{1}{4 \varepsilon}$ for the last inequality and using it in (2.8), we get

$$
\begin{aligned}
& \frac{d}{d t}\left(\|A(t)\|^{2}+\|P h(t)\|^{2}\right)+2\left\|\partial_{x} A(t)\right\|^{2}+m \int_{0}^{l}|h(x, t)|^{4} d x \\
& \quad+k \int_{0}^{l}|A(x, t)|^{4} d x+\gamma m\left\|\partial_{x} h(t)\right\|^{2} \\
& \leq 2 \alpha\|A(t)\|^{2}+C_{2}\left(\gamma, m, \lambda_{1}\right)\|P h(t)\|^{2} .
\end{aligned}
$$

Thanks to (2.9), it is true that

$$
\begin{aligned}
& \|A(t)\|^{2}+\|P h(t)\|^{2} \leq D_{1}(t), \quad \forall t \in R^{+}, \\
& \int_{0}^{t}\left\|\partial_{x} A(t)\right\|^{2} d s, k \int_{0}^{t} \int_{0}^{l}|A(x, t)|^{4} d x d s \leq D_{2}(t),
\end{aligned}
$$


and

$$
\gamma m \int_{0}^{t}\left\|\partial_{x} h(t)\right\|^{2} d s, m \int_{0}^{t} \int_{0}^{l}|h(x, t)|^{4} d x d s \leq D_{2}(t), \quad \forall t \in R^{+},
$$

where $D_{1}(t)=\left[\left\|A_{0}\right\|^{2}+\left\|P h_{0}\right\|^{2}\right] e^{\left(2 \alpha+C_{2}\right) t}, D_{2}(t)=D_{1}(t)+\left\|A_{0}\right\|^{2}+\left\|P h_{0}\right\|^{2}$.

Based on (2.9) and (2.10), we use the standard Faedo-Galerkin method to prove the existence of a global weak solution $[A, h]$ of problem (2.1)-(2.4) with the following properties:

$$
\begin{aligned}
& A \in C\left(0, T ; L^{2}(0, l)\right) \cap L^{2}\left(0, T ; H_{0}^{1}(0, l)\right), \\
& h \in C\left(0, T ; H^{-1}(0, l)\right) \cap L^{2}\left(0, T ; H_{0}^{1}(0, l)\right) .
\end{aligned}
$$

Multiplying (1.2) by $h$ in $L^{2}(0, l)$, we have

$$
\begin{aligned}
\frac{1}{2} \frac{d}{d t}\|h(t)\|^{2}= & -3 m \int_{0}^{l} h^{2}(x, t) \partial_{x} h^{2}(x, t) d x+m \int_{0}^{l}\left|\partial_{x} h(x, t)\right|^{2} d x \\
& +\gamma m \int_{0}^{l} \partial_{x} h(x, t) \partial_{x}^{3} h(x, t) d x-\int_{0}^{l}|A(x, t)|^{2} \partial_{x} h(x, t) \cdot h d x .
\end{aligned}
$$

By Young's inequality, we see that

$$
\begin{aligned}
& \frac{1}{2} \frac{d}{d t}\|h(t)\|^{2}+\gamma m\left\|\partial_{x}^{2} h(t)\right\|^{2} \\
& \quad \leq m\left\|\partial_{x} h(t)\right\|^{2}+\frac{1}{4 m} \int_{0}^{l}|A(x, t)|^{4} d x+\frac{1}{4 m} \int_{0}^{l}|h(x, t)|^{4} d x+\frac{m}{2}\left\|\partial_{x} h(t)\right\|^{2} \\
& \quad \leq \frac{3 m}{2}\left\|\partial_{x} h(t)\right\|^{2}+\frac{1}{4 m} \int_{0}^{l}|A(x, t)|^{4} d x+\frac{1}{4 m} \int_{0}^{l}|h(x, t)|^{4} d x
\end{aligned}
$$

Applying (2.11), integrating inequality $(2.14)$ in $(0, t)$ gives the following estimate:

$$
\begin{aligned}
& \frac{1}{2}\|h(t)\|^{2}+\gamma m \int_{0}^{t}\left\|\partial_{x}^{2} h(s)\right\|^{2} d s \\
& \quad \leq\left(\frac{1}{4 m k}+\frac{1}{4 m^{2}}\right) D_{2}(t)+\frac{3 m}{2} \int_{0}^{t}\left\|\partial_{x} h(s)\right\|^{2} d s+\frac{1}{2}\left\|h_{0}\right\|^{2} \\
& \quad \leq D_{3}(t), \quad \forall t \in[0, T]
\end{aligned}
$$

where $D_{3}(t):=\left(\frac{1}{4 m k}+\frac{1}{4 m^{2}}+\frac{3}{2 \gamma}\right) D_{2}(t)+\frac{1}{2}\left\|h_{0}\right\|^{2}$. Therefore, we deduce

$$
\|h(t)\|^{2}, 2 \gamma m \int_{0}^{t}\left\|\partial_{x}^{2} h(s)\right\|^{2} d s \leq 2 D_{3}(T), \quad \forall t \in[0, T]
$$

Multiplying equation (1.1) by $\partial_{t} A$ in $L^{2}(0, l)$, we see

$$
\begin{gathered}
\left\|\partial_{t} A(t)\right\|^{2}+\frac{d}{d t}\left[\frac{1}{2}\left\|\partial_{x} A(t)\right\|^{2}-\frac{\mu}{2}\|A(t)\|^{2}+\frac{k}{2} \int_{0}^{l}|A(x, t)|^{4} d x\right] \\
=\left(\left\langle A(t), \partial_{t} A(t)\right\rangle, h(t)\right) .
\end{gathered}
$$


Depending on Sobolev's imbedding theorem and estimates (2.11), (2.16), the term on the right-hand side of (2.17) can be estimated as follows:

$$
\begin{aligned}
\left|\left(\left\langle A(t), \partial_{t} A(t)\right\rangle, h(t)\right)\right| & \leq\|A(t)\|_{L^{\infty}(0, l)}\|h(t)\|\left\|\partial_{t} A(t)\right\| \\
& \leq \frac{1}{2}\left\|\partial_{t} A(t)\right\|^{2}+\frac{1}{2}\|A(t)\|_{L^{\infty}(0, l)}^{2}\|h(t)\|^{2} \\
& \leq \frac{1}{2}\left\|\partial_{t} A(t)\right\|^{2}+\frac{l}{2}\left\|\partial_{x} A(t)\right\|^{2}\|h(t)\|^{2} \\
& \leq \frac{1}{2}\left\|\partial_{t} A(t)\right\|^{2}+l D_{3}(t)\left\|\partial_{x} A(t)\right\|^{2}, \quad \forall t \in[0, T] .
\end{aligned}
$$

Thus, according to (2.17),

$$
\begin{aligned}
& \left\|\partial_{t} A(t)\right\|^{2}+\frac{d}{d t}\left[\left\|\partial_{x} A(t)\right\|^{2}-\mu\|A(t)\|^{2}+k \int_{0}^{l}|A(x, t)|^{4} d x\right] \\
& \leq 2 l D_{3}(t)\left\|\partial_{x} A(t)\right\|^{2}, \quad \forall t \in[0, T] .
\end{aligned}
$$

It is easy to obtain the estimate

$$
\int_{0}^{t}\left\|\partial_{s} A(s)\right\|^{2} d s,\left\|\partial_{x} A(t)\right\|^{2} \leq D(T), \quad \forall t \in[0, T],
$$

where

$$
D(T):=2 l D_{3}(T) D_{2}(t)+\alpha D_{1}(T)+\left\|\partial_{x} A_{0}\right\|^{2}-\alpha\left\|A_{0}\right\|^{2}+k \int_{0}^{l}\left|A_{0}(x)\right|^{4} d x
$$

Remark 2.1 If $\lambda_{1}\left(\gamma m-\frac{1}{2 \lambda_{1}^{2}}\right)-\frac{3 m}{2}=r_{0}>0$, the following uniform estimate holds true:

$$
\|A(t)\|^{2}+\|P h(t)\|^{2} \leq\left[\left\|A_{0}\right\|^{2}+\left\|P h_{0}\right\|^{2}\right] e^{-\gamma_{1} t}+\frac{\alpha^{2} l}{2 k \gamma_{1}}
$$

where $\gamma_{1}:=2 \lambda_{1} \min \left\{1, r_{0}\right\}$.

Proof We deduce from (2.6) the inequality

$$
\begin{aligned}
\frac{1}{2} \frac{d}{d t}\|P h(t)\|^{2} & \\
= & -m \int_{0}^{l}|h(x, t)|^{4} d x+m\|h(t)\|^{2}-\gamma m\left\|\partial_{x} h(t)\right\|^{2} \\
& -\int_{0}^{l}\left(|A(x, t)| \partial_{x} h(x, t)\right) P^{2} h(x, t) d x \\
\leq & -m \int_{0}^{l}|h(x, t)|^{4} d x+m\|h(t)\|^{2}-\gamma m\left\|\partial_{x} h(t)\right\|^{2}+\frac{1}{8 m} \int_{0}^{l}|A(x, t)|^{4} d x \\
& +\frac{m \lambda_{1}^{4}}{2} \int_{0}^{l}\left|P^{2} h(x, t)\right|^{4} d x+\frac{1}{2 \lambda_{1}^{2}} \int_{0}^{l}\left|\partial_{x} h(x, t)\right|^{2} d x \\
\leq & -\frac{m}{2} \int_{0}^{l}|h(x, t)|^{4} d x+m\|h(t)\|^{2}-\left(\gamma m-\frac{1}{2 \lambda_{1}^{2}}\right)\left\|\partial_{x} h(t)\right\|^{2}
\end{aligned}
$$




$$
\begin{aligned}
& +\frac{1}{8 m} \int_{0}^{l}|A(x, t)|^{4} d x \\
\leq & m\|h(t)\|^{2}-\left(\gamma m-\frac{1}{2 \lambda_{1}^{2}}\right)\left\|\partial_{x} h(t)\right\|^{2}+\frac{1}{8 m} \int_{0}^{l}|A(x, t)|^{4} d x .
\end{aligned}
$$

From (2.5), we know that

$$
\begin{aligned}
& \frac{1}{2} \frac{d}{d t}\|A(t)\|^{2}+\left\|\partial_{x} A(t)\right\|^{2}+k \int_{0}^{l}|A(x, t)|^{4} d x \\
& \quad \leq \frac{1}{2 m} \int_{0}^{l}|A(x, t)|^{4} d x+\frac{m}{2}\|h(t)\|^{2}+\frac{k}{2} \int_{0}^{l}|A(x, t)|^{4} d x+\frac{\alpha^{2} l}{2 k} .
\end{aligned}
$$

Adding the above two inequalities, we derive

$$
\begin{aligned}
& \frac{1}{2} \frac{d}{d t}\left(\|P h(t)\|^{2}+\|A(t)\|^{2}\right)+\left\|\partial_{x} A(t)\right\|^{2}+k \int_{0}^{l}|A(x, t)|^{4} d x \\
& \quad \leq \frac{3 m}{2}\|h(t)\|^{2}-\left(\gamma m-\frac{1}{2 \lambda_{1}^{2}}\right)\left\|\partial_{x} h(t)\right\|^{2}+k \int_{0}^{l}|A(x, t)|^{4} d x+\frac{\alpha^{2} l}{2 k}
\end{aligned}
$$

that is,

$$
\begin{aligned}
& \frac{1}{2} \frac{d}{d t}\left(\|P h(t)\|^{2}+\|A(t)\|^{2}\right)+\left(\gamma m-\frac{1}{2 \lambda_{1}^{2}}\right)\left\|\partial_{x} h(t)\right\|^{2} \\
& -\frac{3 m}{2}\|h(t)\|^{2}+\left\|\partial_{x} A(t)\right\|^{2} \leq \frac{\alpha^{2} l}{2 k} .
\end{aligned}
$$

Hence

$$
\frac{1}{2} \frac{d}{d t}\left(\|P h(t)\|^{2}+\|A(t)\|^{2}\right)+r_{0}\|h(t)\|^{2}+\left\|\partial_{x} A(t)\right\|^{2} \leq \frac{\alpha^{2} l}{2 k} .
$$

Taking $\gamma_{1}=2 \lambda_{1} \min \left\{1, r_{0}\right\}$, we have

$$
\frac{d}{d t}\left(\|P h(t)\|^{2}+\|A(t)\|^{2}\right)+\gamma_{1}\left(\|P h(t)\|^{2}+\|A(t)\|^{2}\right) \leq \frac{\alpha^{2} l}{2 k} .
$$

We get (2.19) by integrating the last inequality.

\section{Exponential decay of solution}

In this section, we are going to prove the exponential decay of solution.

Proof of Theorem 1.1. Multiplying in $L^{2}(0, l)(2.1)$ by $A,(2.2)$ by $h$, and adding the obtained relations, we get

$$
\begin{gathered}
\frac{1}{2} \frac{d}{d t}\left(\|P h(t)\|^{2}+\|A(t)\|^{2}\right)+\left\|\partial_{x} A(t)\right\|^{2}+k \int_{0}^{l}|A(x, t)|^{4} d x-\alpha\|A(t)\|^{2} \\
\quad+\left(\gamma m-\frac{1}{2 \lambda_{1}^{2}}\right)\left\|\partial_{x} h(t)\right\|^{2}-\frac{3 m}{2}\|h(t)\|^{2}-\frac{5}{8 m} \int_{0}^{l}|A(x, t)|^{4} d x \leq 0,
\end{gathered}
$$


that is,

$$
\frac{1}{2} \frac{d}{d t}\left(\|P h(t)\|^{2}+\|A(t)\|^{2}\right)+d_{0}\|A(t)\|^{2}+\frac{k}{2} \int_{0}^{l}|A(x, t)|^{4} d x+r_{0} \lambda_{1}\|P h(t)\|^{2} \leq 0,
$$

which implies

$$
\frac{1}{2} \frac{d}{d t}\left(\|P h(t)\|^{2}+\|A(t)\|^{2}\right)+\gamma_{0}\left(\|P h(t)\|^{2}+\|A(t)\|^{2}\right)+\frac{k}{2} \int_{0}^{l}|A(x, t)|^{4} d x \leq 0,
$$

where $d_{0}:=\lambda_{1}-\alpha, r_{0}:=\lambda_{1}\left(\gamma m-\frac{1}{2 \lambda_{1}^{2}}\right)-\frac{3 m}{2}$, and $\gamma_{0}=\min \left\{d_{0}, r_{0} \lambda_{1}\right\}$. Hence, we have

$$
\|P h(t)\|^{2}+\|A(t)\|^{2} \leq\left(\left\|P h_{0}\right\|^{2}+\left\|A_{0}\right\|^{2}\right) e^{-2 \gamma_{0} t} .
$$

We conclude from (3.1) that

$$
\begin{aligned}
& \frac{d}{d t}\left(\|P h(t)\|^{2}+\|A(t)\|^{2}\right)+2 d_{0} \lambda_{1}^{-1}\left\|\partial_{x} A(t)\right\|^{2}+2 r_{0} \lambda_{1}^{-1}\left\|\partial_{x} h(t)\right\|^{2} \\
& \quad+k \int_{0}^{l}|A(x, t)|^{4} d x \leq 0 .
\end{aligned}
$$

Integrating this inequality over the interval $(0, t)$ and employing estimate (3.3), we obtain

$$
\frac{d_{0}}{\lambda_{1}} \int_{0}^{t}\left\|\partial_{x} A(\tau)\right\|^{2} d \tau+\frac{r_{0}}{\lambda_{1}} \int_{0}^{t}\left\|\partial_{x} h(\tau)\right\|^{2} d \tau \leq \frac{1}{2}\left(\left\|P h_{0}\right\|^{2}+\left\|A_{0}\right\|^{2}\right), \quad \forall t>0
$$

We can know that if $A_{0}, h_{0} \in H_{0}^{1}(0, l)$ then problem (1.1)-(1.4) has a unique weak solution such that

$$
A, h \in C\left(0, T ; H_{0}^{1}(0, l)\right) \cap L^{2}\left(0, T ; H^{2}(0, l)\right), \quad \forall T>0 .
$$

Taking the inner product of (2.2) with $-\partial_{x}^{2} h$, we have

$$
\frac{d}{d t}\|h(t)\|^{2}+2 \frac{1}{\lambda_{1}}\left(\lambda_{1} \gamma m-m\right)\left\|\partial_{x}^{2} h(t)\right\|^{2} \leq \frac{1}{2 m} \int_{0}^{l}|A(x, t)|^{2} d x .
$$

Besides, we know

$$
\frac{d}{d t}\left(\|P h(t)\|^{2}+\|A(t)\|^{2}\right)+2 d_{0}\|A(t)\|^{2}+k \int_{0}^{l}|A(x, t)|^{4} d x+2 r_{0}\|h(t)\|^{2} \leq 0 .
$$

Let us multiply (3.6) by a positive parameter $\varepsilon_{1}$ and add it with the above inequality

$$
\begin{aligned}
& \frac{d}{d t}\left(\varepsilon_{1}\|h(t)\|^{2}+\|P h(t)\|^{2}+\|A(t)\|^{2}\right)+\frac{2\left(\lambda_{1} \gamma m-m\right) \varepsilon_{1}}{\lambda_{1}}\left\|\partial_{x}^{2} h(t)\right\|^{2} \\
& \quad+\left(2 d_{0}-\frac{\varepsilon_{1}}{2 m}\right)\|A(t)\|^{2}+2 r_{0}\|h(t)\|^{2}+k \int_{0}^{l}|A(x, t)|^{4} d x \leq 0 .
\end{aligned}
$$


Choosing $\varepsilon_{1}=2 m d_{0}$, we obtain the inequality

$$
\begin{aligned}
& \frac{d}{d t} E_{1}(t)+\delta_{1} E_{1}(t)+\frac{2\left(\lambda_{1} \gamma m-m\right) \varepsilon_{1}}{\lambda_{1}}\left\|\partial_{x}^{2} h(t)\right\|^{2}+k \int_{0}^{l}|A(x, t)|^{4} d x \\
& \quad \leq 0, \quad \forall t \geq 0,
\end{aligned}
$$

where

$$
E_{1}(t):=\varepsilon_{1}\|h(t)\|^{2}+\|P h(t)\|^{2}+\|A(t)\|^{2},
$$

and $\delta_{1}:=\min \left\{d_{0}, \lambda_{1} r_{0}, \frac{r_{0}}{\varepsilon_{1}}\right\}$. Then we obtain

$$
E_{1}(t) \leq E_{1}(0) e^{-\delta_{1} t}, \quad \forall t \geq 0 .
$$

Taking the inner product in $L^{2}(0, l)$ of $(2.1)$ with $\partial_{t} A$, we get

$$
\begin{gathered}
\left\|\partial_{t} A(t)\right\|^{2}+\frac{d}{d t}\left(-\frac{\alpha}{2}\|A(t)\|^{2}+\frac{1}{2}\left\|\partial_{x} A(t)\right\|^{2}+\frac{k}{4} \int_{0}^{l}|A(x, t)|^{4} d x\right) \\
\leq\left\|\partial_{t} A(t)\right\|^{2}+\frac{1}{4} \int_{0}^{l}|A(x, t)|^{2}|h(x, t)|^{2} d x .
\end{gathered}
$$

According to [6],

$$
\begin{aligned}
& \frac{d}{d t}\left(-\frac{\alpha}{2}\|A(t)\|^{2}+\frac{1}{2}\left\|\partial_{x} A(t)\right\|^{2}+\frac{k}{4} \int_{0}^{l}|A(x, t)|^{4} d x\right) \\
& \quad \leq \varepsilon_{2} \int_{0}^{l}|A(x, t)|^{4} d x+\varepsilon_{3}\left\|\partial_{x} A(t)\right\|^{2}+\frac{1}{256 \varepsilon_{2}^{2} \varepsilon_{3}}\|h(t)\|^{6} .
\end{aligned}
$$

Multiplying (3.4) by $\frac{1}{2}$ and adding with the inequality, we obtain

$$
\begin{aligned}
& \frac{d}{d t} E_{2}(t)+\frac{d_{0}}{\lambda_{1}}\left\|\partial_{x} A(t)\right\|^{2}+\left(\frac{r_{0}}{\lambda_{1}}-\varepsilon_{3}\right)\left\|\partial_{x} h(t)\right\|^{2}+\left(\frac{k}{2}-\varepsilon_{2}\right) \int_{0}^{l}|A(x, t)|^{4} d x \\
& \quad \leq \frac{1}{256 \varepsilon_{2}^{2} \varepsilon_{3}}\|h(t)\|^{6},
\end{aligned}
$$

where

$$
E_{2}(t):=\frac{(1-\alpha)}{2}\|A(t)\|^{2}+\frac{1}{2}\|P h(t)\|^{2}+\frac{1}{2}\left\|\partial_{x} A(t)\right\|^{2}+\frac{k}{4} \int_{0}^{l}|A(x, t)|^{4} d x .
$$

We choose in the last inequality $\varepsilon_{2}=\frac{k}{4}, \varepsilon_{3}=\frac{r_{0}}{2 \lambda_{1}}$ and obtain the equality

$$
\frac{d}{d t} E_{2}(t)+\delta_{2} E_{2}(t) \leq A_{0}\|A(t)\|^{2}+A_{1}\|h(t)\|^{6}, \quad \forall t \geq 0
$$

where

$$
\delta_{2}:=\min \left\{\frac{2 d_{0}}{\lambda_{1}}, 1,2 r_{0} \lambda_{1}\right\}, \quad A_{0}=\frac{\delta_{2}|1-\alpha|}{2}, \quad A_{1}=\frac{1}{256 \varepsilon_{2}^{2} \varepsilon_{3}} .
$$


Using estimate (3.8), we have

$$
\frac{d}{d t} E_{2}(t)+\delta_{2} E_{2}(t) \leq A_{2} e^{-\delta_{1} t}
$$

where $A_{2}=A_{1} E_{1}^{3}(0)+A_{0} E_{1}(0)$. Integrating (3.9) and by Gronwall's inequality, we get

$$
E_{2}(t) \leq \frac{A_{2}}{\delta_{1}} e^{-\delta_{2} t}
$$

Combining with (3.3), we have

$$
\left\|\partial_{x} A(t)\right\|^{2} \leq \frac{2 A_{2}}{\delta_{1}} e^{-\delta_{2} t}+R_{0} e^{-2 \gamma_{0} t} .
$$

Multiplying (2.2) by $\partial_{t} h$, we obtain

$$
\begin{aligned}
\left\|P \partial_{t} h(t)\right\|^{2}+\frac{d}{d t}\left(-\frac{m}{2}\|h(t)\|^{2}+\frac{\gamma m}{2}\left\|\partial_{x} h(t)\right\|^{2}\right) \\
\leq-\frac{m}{4} \frac{d}{d t} \int_{0}^{l}|h(x . t)|^{4} d x \\
\quad+\frac{1}{4} \int_{0}^{l}\left[P\left(|A(x, t)| \partial_{x} h(x, t)\right)\right]^{2} d x+\left\|P \partial_{t} h(t)\right\|^{2} .
\end{aligned}
$$

Adding $\frac{m}{2}$ (3.6) to (3.12), we get

$$
\begin{aligned}
& \frac{d}{d t}\left(\frac{\gamma m}{2}\left\|\partial_{x} h(t)\right\|^{2}+\frac{m}{4} \int_{0}^{l}|h(x . t)|^{4} d x\right)+2\left(\lambda_{1} \gamma m-m\right)\left\|\partial_{x} h(t)\right\|^{2} \\
& \quad \leq \frac{1}{2 m} \int_{0}^{l}|A(x, t)|^{2} d x+\frac{l}{4 \lambda_{1}}\left\|\partial_{x} A(t)\right\|^{2}\left\|\partial_{x} h(t)\right\|^{2}
\end{aligned}
$$

thus

$$
\begin{aligned}
& \frac{d}{d t}\left(\frac{\gamma m}{2}\left\|\partial_{x} h(t)\right\|^{2}+\frac{m}{4} \int_{0}^{l}|h(x . t)|^{4} d x\right)+\left[2\left(\lambda_{1} \gamma m-m\right)-\frac{l}{4 \lambda_{1}}\right]\left\|\partial_{x} h(t)\right\|^{2} \\
& \quad \leq \frac{1}{2 m} \int_{0}^{l}|A(x, t)|^{2} d x .
\end{aligned}
$$

Note that

$$
\int_{0}^{l}|h(x . t)|^{4} d x \leq\|h(t)\|_{\infty}^{2}\|h(t)\|^{2} \leq l\left\|\partial_{x} h(t)\right\|^{2}\|h(t)\|^{2} \leq c\left\|\partial_{x} h(t)\right\|^{2} .
$$

Hence, we know that

$$
\begin{aligned}
& \frac{d}{d t}\left(\frac{\gamma m}{2}\left\|\partial_{x} h(t)\right\|^{2}+\frac{m}{4} \int_{0}^{l}|h(x . t)|^{4} d x\right) \\
& \quad+c\left(\frac{\gamma m}{2}\left\|\partial_{x} h(t)\right\|^{2}+\frac{m}{4} \int_{0}^{l}|h(x . t)|^{4} d x\right) \leq \frac{E_{2}(0)}{2 m} e^{-\delta_{1} t}
\end{aligned}
$$


Finally, we integrate (3.13) and get

$$
\frac{\gamma m}{2}\left\|\partial_{x} h(t)\right\|^{2}+\frac{m}{4} \int_{0}^{l}|h(x . t)|^{4} d x \leq c_{1} e^{-c t}
$$

where $c_{1}=-\frac{E_{2}(0)}{2 m \delta_{1}} e^{-\delta_{1} t}+\frac{m}{2}\left\|\partial_{x} h_{0}\right\|^{2}+\frac{m}{4} \int_{0}^{l}\left|h_{0}\right|^{4} d x-\frac{E_{2}(0)}{2 m \delta_{1}}$. The theorem is true.

\section{Continuous dependence results}

Assume that $[\tilde{A}, \tilde{h}]$ is the weak solution of the problem

$$
\begin{aligned}
& \partial_{t} \tilde{A}-\alpha \tilde{A}-\partial_{x}^{2} \tilde{A}+\tilde{k}|\tilde{A}|^{2} \tilde{A}=\tilde{A} \tilde{h}, \quad x \in(0, l), t>0, \\
& \partial_{t} \tilde{h}+|\tilde{A}| \partial_{x} \tilde{h}=m \partial_{x}^{2} \tilde{\mu}, \quad x \in(0, l), t>0, \\
& \tilde{A}(x, 0)=A_{0}(x), \quad \tilde{h}(x, 0)=h_{0}(x), \quad x \in(0, l), \\
& \tilde{A}(0, t)=\tilde{A}(l, t)=\overrightarrow{0}, \quad \partial_{x} \tilde{h}(0, t)=\partial_{x}^{3} \tilde{h}(0, t)=\partial_{x} \tilde{h}(l, t)=\partial_{x}^{3} \tilde{h}(l, t)=0, \quad t>0,
\end{aligned}
$$

where $\tilde{\mu}=f^{\prime}(\tilde{h})-\gamma \partial_{x}^{2} \tilde{h}, f^{\prime}(\tilde{h})=\tilde{h}^{3}-\tilde{h}$.

Theorem 4.1 Assume that $[A, h]$ is a solution of problem $(1.1)-(1.4)$ and $[\tilde{A}, \tilde{h}]$ is a solution of problem (4.1)-(4.4). Let $[a, H]=[A-\tilde{A}, h-\tilde{h}]$, we have

$$
\|P H(t)\|^{2}+\|a(t)\|^{2} \leq q_{0} e^{-\int_{0}^{t} R_{1}(s) d s} \int_{0}^{t}\left\|\partial_{x} A(s)\right\|^{4} d s
$$

where $q_{0}=\frac{2 k_{1}^{\frac{4}{3}} C_{0}^{4}}{\left(k_{2} b_{0}\right)^{\frac{1}{3}}}+\frac{C_{0}^{4}}{4 m^{3} \lambda_{1}^{4} \gamma^{2}}$

$$
R_{1}(t)=2 C(\gamma, m)+2 C_{1}(\gamma, m)+4 l\|h(t)\|^{2}+2 \alpha+\frac{l}{\lambda_{1}^{2} m}\left\|\partial_{x}^{2} \tilde{h}(t)\right\|^{2}+l\left\|\partial_{x}^{2} \tilde{A}(t)\right\|^{2}
$$

Proof Note that $[a, H]=[A-\tilde{A}, h-\tilde{h}]$ is a solution of the following problem:

$$
\begin{aligned}
& \partial_{t} a-\alpha a-\partial_{x}^{2} a+k|A|^{2} A-\tilde{k}|\tilde{A}|^{2} \tilde{A}=A h-\tilde{A} \tilde{h}, \quad x \in(0, l), t>0, \\
& P^{2} \partial_{t} H-m H-\gamma m \partial_{x}^{2} H \\
& \quad=-m\left(h^{3}-\tilde{h}^{3}\right)+P^{2}\left(|\tilde{A}| \partial_{x} \tilde{h}\right)-P^{2}\left(|A| \partial_{x} h\right), \quad x \in(0, l), t>0, \\
& a(x, 0)=\overrightarrow{0}, \quad H(x, 0)=0, \quad x \in(0, l), \\
& a(0, t)=a(l, t)=\overrightarrow{0}, \quad \partial_{x} H(0, t)=\partial_{x} H(0, t), \quad x \in(0, l), t>0 .
\end{aligned}
$$

By

$$
A h-\tilde{A} \tilde{h}=A h-\tilde{A} h+\tilde{A} h-\tilde{A} \tilde{h}=a h+\tilde{A} H
$$

and

$$
P^{2}\left(|\tilde{A}| \partial_{x} \tilde{h}\right)-P^{2}\left(|A| \partial_{x} h\right)=P^{2}\left(|\tilde{A}| \partial_{x} \tilde{h}-|A| \partial_{x} h\right)
$$




$$
\begin{aligned}
& =P^{2}\left(|\tilde{A}| \partial_{x} \tilde{h}-|A| \partial_{x} \tilde{h}+|A| \partial_{x} \tilde{h}-|A| \partial_{x} h\right) \\
& =P^{2}\left(-|A| \partial_{x} H-\partial_{x} \tilde{h}(|A|-|\tilde{A}|)\right),
\end{aligned}
$$

we see that $[a, H]$ satisfies the following system:

$$
\begin{aligned}
& \partial_{t} a-\alpha a-\partial_{x}^{2} a+k_{2}\left(|A|^{2} A-|\tilde{A}|^{2} \tilde{A}\right)=k_{1}|A|^{2} A+a h+\tilde{A} H, \\
& P^{2} \partial_{t} H-m H-\gamma m \partial_{x}^{2} H \\
& \quad=-m\left(h^{3}-\tilde{h}^{3}\right)-P^{2}\left(|A| \partial_{x} H+\partial_{x} \tilde{h}(|A|-|\tilde{A}|)\right),
\end{aligned}
$$

where $k_{2}=\tilde{k}, k_{1}=\tilde{k}-k$.

On the other hand, we know that

$$
\left(|A(t)|^{2} A(t)-|\tilde{A}(t)|^{2} \tilde{A}(t), A(t)-\tilde{A}(t)\right) \geq b_{0} \int_{0}^{l}|a(x, t)|^{4} d x .
$$

Multiplying (4.7) by $a$ and using inequality (4.9), we obtain

$$
\begin{aligned}
\frac{1}{2} \frac{d}{d t}\|a(t)\|^{2}-\alpha\|a(t)\|^{2}+\left\|\partial_{x} a(t)\right\|^{2}+k_{2} b_{0} \int_{0}^{l}|a(x, t)|^{4} d x \\
\leq k_{1} \int_{0}^{l}|A(x, t)|^{2}\langle A(x, t), a(x, t)\rangle d x+\int_{0}^{l}|a(x, t)|^{2} h(x, t) d x \\
\quad+\int_{0}^{l}\langle\tilde{A}(x, t), a(x, t)\rangle H(x, t) d x .
\end{aligned}
$$

We are going to estimate the first integral on the right-hand side of (4.10) by the Nirenberg inequality as follows:

$$
\begin{aligned}
\left.\left|\int_{0}^{l}\right| a(x, t)\right|^{2} h(x, t) d x \mid & \leq\|a(t)\|_{\infty}^{2} \int_{0}^{l}|h(x, t)| d x \\
& \leq \sqrt{l}\|a(t)\|_{\infty}^{2}\|h(t)\| \\
& \leq 2 \sqrt{l}\|a(t)\|\left\|\partial_{x} a(t)\right\|\|h(t)\| \\
& \leq \frac{1}{2}\left\|\partial_{x} a(t)\right\|^{2}+2 l\|a(t)\|^{2}\|h(t)\|^{2} .
\end{aligned}
$$

We can infer from the Nirenberg inequality and the Friedrichs inequality that the following estimate of the second term on the right-hand side of (4.10) is true:

$$
\begin{aligned}
& \mid \int_{0}^{l}\langle\tilde{A}(x, t), a(x, t)|H(x, t) d x| \\
& \quad \leq \int_{0}^{l}|\tilde{A}(x, t)\|a(x, t)\| H(x, t)| d x \\
& \quad \leq\|\tilde{A}(t)\|_{\infty}\|a(t)\|\|H(t)\| \\
& \quad \leq \frac{1}{2}\|\tilde{A}(t)\|_{\infty}^{2}\|a(t)\|^{2}+\frac{1}{2}\|H(t)\|^{2} \\
& \quad \leq \frac{l}{2}\left\|\partial_{x} \tilde{A}(t)\right\|^{2}\|a(t)\|^{2}+\varepsilon_{2}\left\|\partial_{x} H(t)\right\|^{2}+C_{1}\left(\varepsilon_{2}\right)\|P H(t)\|^{2} .
\end{aligned}
$$


Employing Young's inequality and Sobolev's inequality, we have

$$
\begin{aligned}
& \left.\left|k_{1} \int_{0}^{l}\right| A(x, t)\right|^{2}|A(x, t), a(x, t)\rangle d x \mid \\
& \quad \leq k_{1} \int_{0}^{l}|A(x, t)|^{3}|a(x, t)| d x \\
& \quad \leq \frac{k_{1}^{\frac{4}{3}}}{\left(k_{2} b_{0}\right)^{\frac{1}{3}}} \int_{0}^{l}|A(x, t)|^{4}+k_{2} b_{0} \int_{0}^{l}|a(x, t)|^{4} d x \\
& \quad \leq \frac{k_{1}^{3} C_{0}^{4}}{\left(k_{2} b_{0}\right)^{\frac{1}{3}}}\left\|\partial_{x} A(t)\right\|^{4} d x+k_{2} b_{0} \int_{0}^{l}|a(x, t)|^{4} d x .
\end{aligned}
$$

Then employing the last inequality and (4.11), (4.12), we deduce the following inequality from (4.10):

$$
\begin{aligned}
\frac{1}{2} \frac{d}{d t}\|a(t)\|^{2}+\left\|\partial_{x} a(t)\right\|^{2}+k_{2} b_{0} \int_{0}^{l}|a(x, t)|^{4} d x \\
\leq \alpha\|a(t)\|^{2}+\frac{k_{1}^{\frac{4}{3}} C_{0}^{4}}{\left(k_{2} b_{0}\right)^{\frac{1}{3}}}\left\|\partial_{x} A(t)\right\|^{4}+2 l\|h(t)\|^{2}\|a(t)\|^{2}+\frac{l}{2}\left\|\partial_{x} \tilde{A}(t)\right\|^{2}\|a(t)\|^{2} \\
\quad+\varepsilon\left\|\partial_{x} H(t)\right\|^{2}+C(\varepsilon)\|P H(t)\|^{2} .
\end{aligned}
$$

Multiplying (4.8) in $L^{2}(0, l)$ by $H$, we get

$$
\begin{aligned}
& \frac{1}{2} \frac{d}{d t}\|P H(t)\|^{2}-m\|H(t)\|^{2}+\gamma m\left\|\partial_{x} H(t)\right\|^{2} \\
& \quad=-m\left(h^{3}-\tilde{h}^{3}, H\right)+\left(|A| \partial_{x} H+\partial_{x} \tilde{h}(|A|-|\tilde{A}|),-P^{2} H\right)
\end{aligned}
$$

and

$$
\begin{aligned}
&\left(|A| \partial_{x} h+\partial_{x} \tilde{h}(|A|-|\tilde{A}|),-P^{2} H\right) \\
&=-\int_{0}^{l}|A| \partial_{x} H P^{2} H d x-\int_{0}^{l} \partial_{x} \tilde{h}(|A|-|\tilde{A}|) P^{2} H d x \\
& \leq \frac{1}{8 m^{3} \lambda_{1}^{4} \gamma^{2}} \int_{0}^{l}|A(x, t)|^{4} d x+m \lambda_{1}^{4} \int_{0}^{l}\left|P^{2} H(t)\right|^{4} d x+\frac{\gamma m}{2} \int_{0}^{l}\left|\partial_{x} H(t)\right|^{2} d x \\
&+\frac{l}{2 \lambda_{1}^{2} m}\left\|\partial_{x}^{2} \tilde{h}(t)\right\|^{2}\|a(t)\|^{2}+\frac{\lambda_{1}^{2} m}{2} \int_{0}^{l}\left|P^{2} H(x, t)\right|^{2} d x \\
& \leq m \int_{0}^{l}|H(x, t)|^{4} d x+\frac{l}{2 \lambda_{1}^{2} m}\left\|\partial_{x}^{2} \tilde{h}(t)\right\|^{2}\|a(t)\|^{2}+\frac{m}{2} \int_{0}^{l}|H(x, t)|^{2} d x \\
&+\frac{\gamma m}{2} \int_{0}^{l}\left|\partial_{x} H(x, t)\right|^{2} d x+\frac{1}{8 m^{3} \lambda_{1}^{4} \gamma^{2}} \int_{0}^{l}|A(x, t)|^{4} d x \\
& \leq m \int_{0}^{l}|H(x, t)|^{4} d x+\frac{l}{2 \lambda_{1}^{2} m}\left\|\partial_{x}^{2} \tilde{h}(t)\right\|^{2}\|a(t)\|^{2}+\frac{5 \gamma m}{8}\left\|\partial_{x} H(t)\right\|^{2} \\
&+C_{1}(\gamma, m)\|P H(t)\|^{2}+\frac{1}{8 m^{3} \lambda_{1}^{4} \gamma^{2}} \int_{0}^{l}|A(x, t)|^{4} d x .
\end{aligned}
$$


Employing Sobolev's imbedding theorem and the Nirenberg inequality, we get

$$
\begin{aligned}
& \left(h^{3}-\tilde{h}^{3}, H\right)=\int_{0}^{l} H^{2}\left(h^{2}+h \tilde{h}+\tilde{h}^{2}\right) d x \geq \int_{0}^{l} H^{4} d x, \\
& |m|\|H(t)\|^{2} \leq \frac{\gamma m}{4}\left\|\partial_{x} H(t)\right\|^{2}+C_{1}(m, \gamma)\|P H(t)\|^{2} .
\end{aligned}
$$

Using (4.15), (4.16), we have

$$
\begin{aligned}
\frac{1}{2} \frac{d}{d t}\|P H(t)\|^{2}+\frac{\gamma m}{8}\left\|\partial_{x} H(t)\right\|^{2} \\
\leq C_{1}(\gamma, m)\|P H(t)\|^{2} \\
\quad+\frac{l}{2 \lambda_{1}^{2} m}\left\|\partial_{x}^{2} \tilde{h}(t)\right\|^{2}\|a(t)\|^{2}+\frac{1}{8 m^{3} \lambda_{1}^{4} \gamma^{2}} \int_{0}^{l}|A(x, t)|^{4} d x .
\end{aligned}
$$

Taking $\varepsilon=\frac{\gamma m}{8}$ in (4.13) and adding it to (4.17), we get

$$
\begin{aligned}
& \frac{d}{d t}\left(\|P H(t)\|^{2}+\|a(t)\|^{2}\right) \\
& \quad \leq\left(\frac{2 k_{1}^{\frac{4}{3}} C_{0}^{4}}{\left(k_{2} b_{0}\right)^{\frac{1}{3}}}+\frac{C_{0}^{4}}{4 m^{3} \lambda_{1}^{4} \gamma^{2}}\right)\left\|\partial_{x} A(t)\right\|^{4}+R_{1}(t)\left(\|P H(t)\|^{2}+\|a(t)\|^{2}\right),
\end{aligned}
$$

where

$$
R_{1}(t)=2 C(\gamma, m)+2 C_{1}(\gamma, m)+4 l\|h(t)\|^{2}+2 \alpha+\frac{l}{\lambda_{1}^{2} m}\left\|\partial_{x}^{2} \tilde{h}(t)\right\|^{2}+l\left\|\partial_{x}^{2} \tilde{A}(t)\right\|^{2} .
$$

From (4.18) we derive the estimate

$$
\|P H(t)\|^{2}+\|a(t)\|^{2} \leq q_{0} e^{-\int_{0}^{t} R_{1}(s) d s} \int_{0}^{t}\left\|\partial_{x} A(s)\right\|^{4} d s,
$$

where $q_{0}=\frac{2 k_{1}^{\frac{4}{3}} C_{0}^{4}}{\left(k_{2} b_{0}\right)^{\frac{1}{3}}}+\frac{C_{0}^{4}}{4 m^{3} \lambda_{1}^{4} \gamma^{2}}$. The proof is complete.

\section{Acknowledgements}

Not applicable.

\section{Funding}

This work is supported by the Jilin Scientific and Technological Development Program [number 20170101143JC].

Availability of data and materials

Not applicable.

\section{Competing interests}

The authors declare that they have no competing interests.

Authors' contributions

All authors contributed equally to the manuscript and read and approved the final manuscript.

\section{Publisher's Note}

Springer Nature remains neutral with regard to jurisdictional claims in published maps and institutional affiliations. Received: 31 December 2018 Accepted: 8 March 2019 Published online: 15 March 2019 
References

1. Cahn, J.W., Novick-Cohen, A.: Evolution equations for phase separation and ordering in binary alloys. J. Stat. Phys. 76 877-909 (1994)

2. Boldrini, J.L., da Silva, P.N.: A generalized solution to a Cahn-Hilliard/Allen-Cahn system. Electron. J. Differ. Equ. 2004, 126 (2004)

3. Liu, A., Liu, C.: The Cauchy problem for the degenerate convective Cahn-Hilliard equation. Rocky Mt. J. Math. 48 , 2595-2623 (2018)

4. Liu, C., Tang, H.: Existence of periodic solution for a Cahn-Hilliard/Allen-Cahn equation in two space dimensions. Evol. Equ. Control Theory 6, 219-237 (2017)

5. Miranville, A., Saoud, W., Talhouk, R.: Asymptotic behavior of a model for order-disorder and phase separation. Asymptot. Anal. 103, 57-76 (2017)

6. Çelebi, O.A., Kalantarov, V.K.: Decay of solutions and structural stability for the coupled Kuramoto-Sivashinsky-Ginzburg-Landau equations. Appl. Anal. 94, 2342-2354 (2015)

7. Çelebi, O.A., Gür, Ş., Kalantarov, V.K.: Structural stability and decay estimate for marine riser equations. Math. Comput. Model. 54, 3182-3188 (2011)

8. Liu, A., Liu, C.: Cauchy problem for a sixth order Cahn-Hilliard type equation with inertial term. Evol. Equ. Control Theory 4, 315-324 (2015)

9. Liu, C., Wang, J.: Some properties of solutions for a sixth order Cahn-Hilliard type equation with inertial term. Appl. Anal. 97, 2332-2348 (2018)

10. Kalantarov, V.K.: Global solution of coupled Kuramoto-Sivashinsky and Ginzburg-Landau equations. In: Rozhkovskaya, T. (ed.) Nonlinear Problems in Mathematical Physics and Related Topics, II. Int. Math. Ser. (N.Y.), pp. 213-227. Kluwer/Plenum, New York (2002)

\section{Submit your manuscript to a SpringerOpen ${ }^{\circ}$ journal and benefit from:}

- Convenient online submission

Rigorous peer review

- Open access: articles freely available online

- High visibility within the field

- Retaining the copyright to your article 